\title{
New hadronic spectroscopy
}

\section{R. FACCINI}

Dipartimento di Fisica, Università di Roma "La Sapienza" and INFN, Sezione di Roma Piazzale A. Moro 2, I-00185 Rome, Italy

(ricevuto l' 8 Ottobre 2010; pubblicato online il 15 Febbraio 2011)

\begin{abstract}
Summary. - In the past few years the field of hadron spectroscopy has seen renewed interest due to the pubblication, initially mostly from $B$-Factories, of evidences of states that do not match regular spectroscopy, but are rather candidates for bound states with additional quarks or gluons (four quarks for tetraquarks and molecules and two quarks and gluons for hybrids). A huge effort in understanding the nature of this new states and in building a new spectroscopy is ongoing. This paper reviews the experimental and theoretical state of the art on heavy quarkonium exotic spectroscopy, with particular attention on the steps towards a global picture.
\end{abstract}

PACS 13.25.Hw - Decays of bottom mesons.

PACS 14.40.Nd - Bottom mesons $(|B|>0)$.

It is now almost seven years since the first heavy quarkonium exotic state has been observed and the number of observed such states has significantly increased. In order to explain all these states one needs to go beyond the assumption that mesons can only be made of a quark and an anti-quark and consider the possibility to be observing bound states of four quarks or two quarks and valence gluons. These novel states of aggregation are not a new idea and it was indeed already in the foundations of the quark model [1]. Also, the hypothesis that the mesons of the scalar nonet are actually tetraquarks can be dated back to the '70s [2]. But only now, with the presence of several exotic candidates with heavy quarks the possibily to have a global picture is becoming real.

The path towards such global picture comprises several steps. First all relevant experimental observations need be considered. Figure 1, from ref. [3], summarizes such observations in the charmonium sector, the richest of exotic candidates. Next, a complete set of theoretical predictions and a systematic experimental search is needed. The work in the theoretical path is progressing although strong interactions are extremely hard to compute, even in presence of heavy quarks, and therefore predictions are extremely hard and uncertain. Finally, additional states predicted by the theoretical models need to be searched for. This paper sketches the path towards this last goal.

The status of the experimental observations is extremely fragmented. Pictorial summaries, separated by production mechanisms, are in figs. 2-4. The exotic states have been observed always in only one production mechanism and often in only one final 

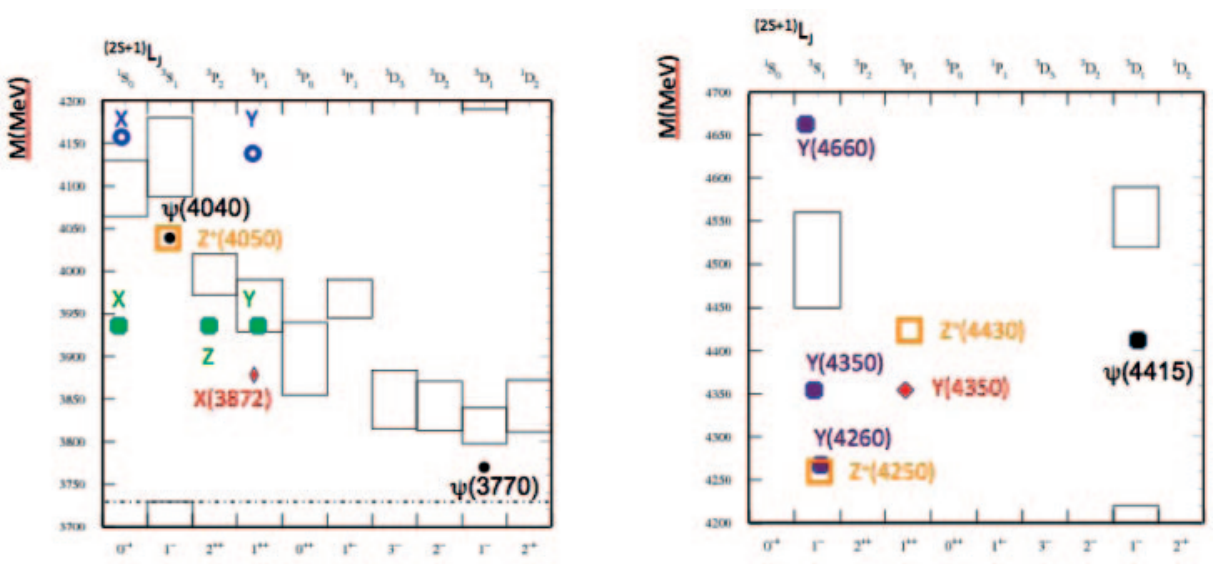

Fig. 1. - Observed states with hidden charm above the open charm threshold. The theory predictions are according to the potential models described in ref. [4].

state. None of the states has been searched systematically in all final states. Sometimes the analysis of a given final state for a given production mechanism is missing, but often it has either been performed only in a limited mass range or the invariant mass spectrum has been published without a fit to the possible new state. This is mostly due to the fact that some of the analyses did not show a significant signal themselves and they were published before a new state was observed. As an example, the invariant mass spectra of a charmonium and a photon in refs. $[5,6]$ are focussed on the $X(3872)$ region and no information is available outside it. On the other side in ref. [7] the $J / \psi \eta$ invariant mass spectrum is published, but not fitted for all the possible new states. Whatever the cause is, the critical point is that building a global picture requires having either an observation or a limit in each final state for each candidate new state: limits allow to quantify the level at which a decay mode has not been observed and can show whether a signal

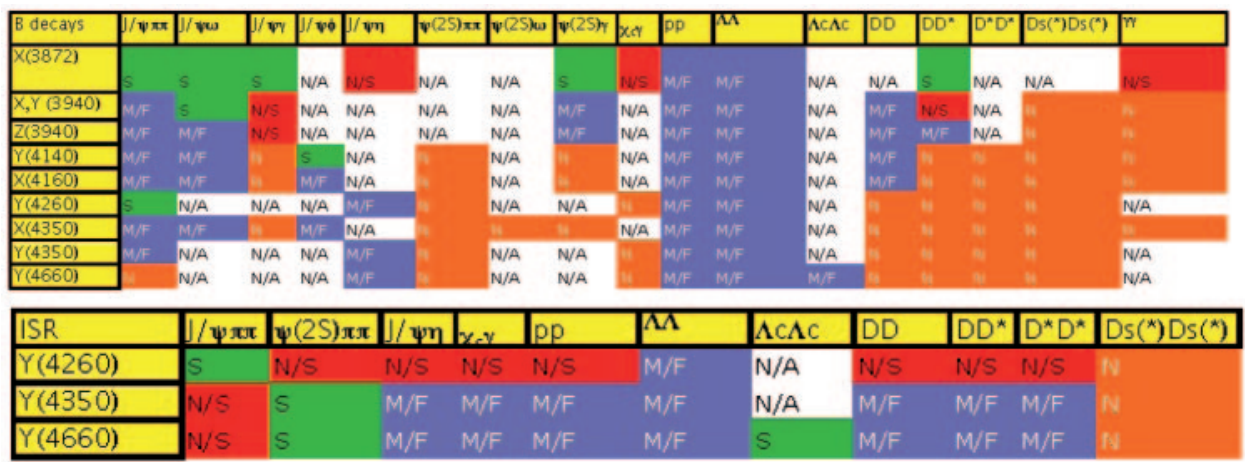

Fig. 2. - Status of the searches of the new states in the processes $B \rightarrow X K$ (top) and $e^{+} e^{-} \rightarrow$ $X \gamma_{I S R}, X \rightarrow f$, for several final states $f$. Final states where each exotic state was observed ("S") or excluded ("N/S") are indicated. A final state is marked as "N" if the analysis has not been performed in a given mass range and with "M/F" if the spectra are published but a fit to a given state has not been performed. Finally "N/A" indicates that quantum numbers forbid the decay and "N/F" if an analysis is experimentally too challenging. 


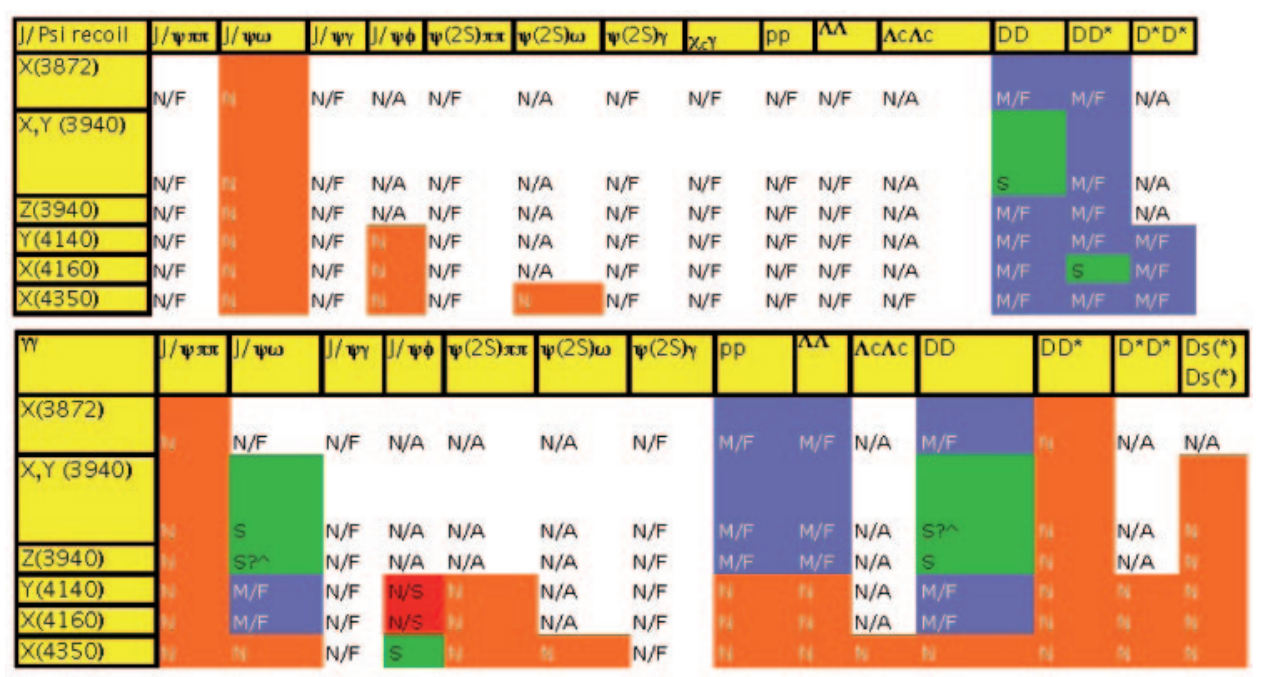

Fig. 3. - Status of the searches of the new states in collisions $e^{+} e^{-} \rightarrow X J / \psi$ (top) and $\gamma \gamma \rightarrow X$ (bottom), $X \rightarrow f$, for several final states $f$. Symbols are explained in the caption of fig. 2.

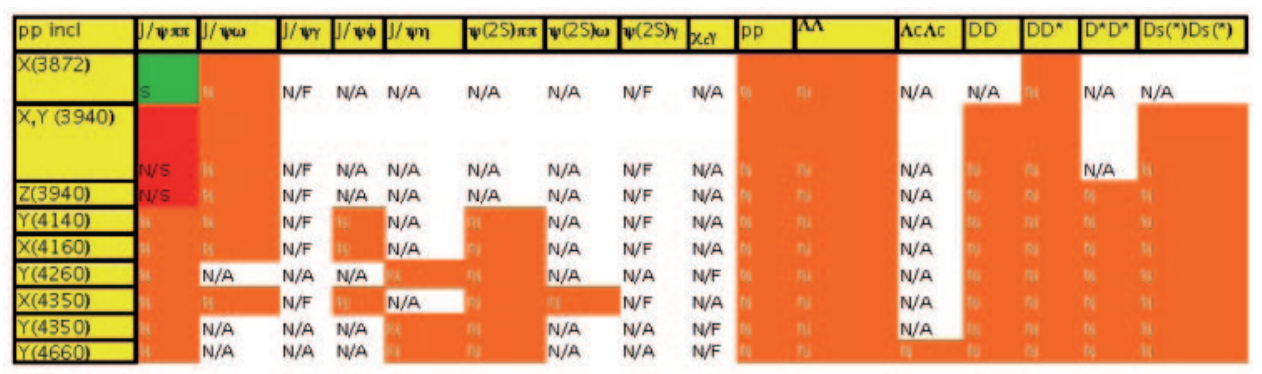

\begin{tabular}{|c|c|c|c|c|c|c|c|c|}
\hline B decays & $1 / 14 \pi$ & 1/14س & $4(25) \pi$ & $125 \mathrm{~s}$ & K.TI & $D D$ & DD* & $D * 0 *$ \\
\hline $2+(3870)$ & $M / F$ & Nis & $M F$ & B & $M / F$ & $\mathrm{~B}$ & B. & N'A \\
\hline$Z+(3940)$ & M/F & B & $W F$ & $\mathrm{~N}$ & $M^{\prime} F$ & N & $\mathrm{H}$ & $N^{\prime A}$ \\
\hline$Z+(4050)$ & M/F & $\mathrm{N}$ & $M F$ & $N$ & S & $\mathrm{N}$ & $\mathrm{N}$ & $M F$ \\
\hline$z+(4140)$ & $M V F$ & $\mathrm{H}$ & $M F$ & $\mathrm{~N}$ & $M^{\prime} F$ & N & $\mathrm{N}$ & $M F$ \\
\hline$z+(4250)$ & MF & N & $M F$ & $\mathrm{~N}$ & s & N & $\mathrm{N}$ & $M F$ \\
\hline$Z+(4350)$ & MF & A & $M F$ & $\mathrm{~N}$ & WF & $\mathrm{M}$ & A & $W^{\prime} F$ \\
\hline$Z+(4430)$ & N's & A & $\mathrm{s}$ & $\mathrm{N}$ & MF & $\mathrm{N}$ & $\mathrm{N}$ & $M F$ \\
\hline $2+(4660)$ & M/F & M & $M F$ & $\mathrm{~N}$ & $M F$ & N & N & $M F$ \\
\hline
\end{tabular}

Fig. 4. - Status of the inclusive searches of the new neutral states in $p \bar{p}$ collisions (top) and of the new charged states (bottom) for several final states. Symbols are explained in the caption of fig. 2 . 
could have been observed in a final state or the efficiency, the branching fraction or the background level would make it unobservable. It is also worth remarking that although finding new decay modes of these states might not necessarily cast light on the nature of these particles, they would in any case concur to the evidence of the existence of the states that suffer from lack of statistics.

Looking into this "observational" tables in detail, $B$ decays (top of fig. 2) are the most studied, but there is a significant amount of missing fits ("M/F"). Particularly severe is the lack of analysis of the baryonic spectra since baryonic decays are a signature of tetraquark states. Some other modes have never been studied, mostly because the number of expected events is very low. Nonetheless the study of $B \rightarrow \psi(2 S) \pi \pi K$ decays should be relatively clean, while $D^{*} \bar{D}^{*}$ and above all $D_{s}^{(*)} \bar{D}_{s}^{(*)}$ suffer from the low branching fractions of the observed states.

States produced in conjunction with an initial state radiation (ISR) photon (bottom of fig. 2) have an unambigous $J^{C P}$ assignment and therefore fewer analyses are needed to establish their properties. Nonetheless it is striking to see that a large fraction of analyses have been carried out exclusively for the first observed exotic state, the $Y(4260)$. It can also be noticed that no search is published involving $D_{s}^{(*)}$ mesons: while the efficiency is expected to be very low, background should be low as well and surprises can always arise. Finally the $Y(4660)$ has been the object of one of the combined analyses we are advocating here [8]: two states apparently different, observed in $\psi(2 S)$ and $\Lambda_{c} \bar{\Lambda}_{c}$ final states, if fitted under the same ansatz were found to be consistent with being the same and interesting ratios of branching fractions were measured.

On the recoil of a $J / \psi$ and in $\gamma \gamma$ interactions (fig. 3) only $C=+$ neutral states can be observed. This restricts the number of final states of interest. Also, the low multiplicity of these decays and the large missing momentum in the case of $\gamma \gamma$ decays makes these analyses experimentally challenging. On the other side $C=+$ states are the least known and reinforcing the evidence of the signals would help. It is also interesting to notice that, mostly due to statistics, the recoil to any other particle but the $J / \psi$ has not been investigated. Given the selection rules the recoil to $\chi_{c 0}$ or $\chi_{c 2}$ would be very interesting.

Searches at hadron colliders (top of fig. 4) have the advantage to have very large samples. On the other side backgrounds are very high and therefore final states with too high multiplicity and above all with neutral particles are not at reach. Finally, it is hard to extract information on production cross sections and therefore branching fractions. Nonetheless, it is clear that a systematic search in $p \bar{p}$ collisions is likely to clarify the picture significantly and it is a pity that it is still missing. This holds also and in particular for the charged states - for instance the longly debated $Z^{+}(4430)$ should give a large signal in the $J / \psi \pi^{+}$spectrum - and in the bottomonium - where no other experiment is in the position of studying inclusive $\Upsilon(n S) \pi^{+} \pi^{-}$and $\Upsilon(n S) \pi^{ \pm}$spectra.

Concerning the searches of charged exotic states, the most striking signature of states made of more than two quarks, very few searches have been conducted in $B$ decays. We believe that for each exotic neutral spectrum the corresponding charged state should be searched for completeness and, as shown in the bottom of fig. 4 only five combinations of final states and exotic states has been searched for. As an example, no information has been extracted from refs. [9,10] on the charged partner of the $X(3872), Z(3870)$ in our table, which has long been pointed out as a critical state to search for. Moreover among the four quark bound states there must be states that contain a single $s$ quark, their mostly distinctive signature being a charmonium plus a charged kaon. These states could be searched in $B$ decays in association with a $s \bar{s}$ state or inclusively in $p \bar{p}$ collisions. 
Concerning the bottomonium states, the searches are extremely limited and there is no confirmed evidence of exotic states yet $[11,12]$. The search potential of $B$-Factories is limited by the fact that bottomonium at masses higher than the open bottom threshold can only be produced in conjunction with initial state radiation and that in addition the accessible mass range is full of threshold openings. This restricts the search to $J^{P C}=1^{--}$ states with significant signatures in exclusive final states. Besides the lack of information about most of the possible final states, the real contribution could come from hadron colliders, where the search could be inclusive over $\Upsilon(n S) X$ final states (where $X$ is made of charged tracks) and extended to exotic states with open bottom, eventually even with open charm.

In conclusion, seven years of discoveries of exotic particles need to be followed by a systematic study of the possible new spectroscopy to be able to give a global and definite picture. To this aim still a lot can be extracted from existing data of $B$-Factories and Tevatron. Nonetheless in the present generation of experiments statistics is extremely low and we will not be able to have a convincing picture until the results of ultra-high intensity machines, LHC and the Super Flavour Factories (SuperB and/or SuperBelle), will be available.

\section{REFERENCES}

[1] Gell-Mann M., Phys. Lett., 8 (1964) 214.

[2] Jaffe R. L., Phys. Rev. D, 15 (1977) 267.

[3] Drenska N., Faccini R., Piccinini F., Polosa A. D., Renga F. et al., Riv. Nuovo Cimento, 33 (2010) 633.

[4] Brambilla N. et al., hep-ph/0412158 preprint (2004).

[5] Aubert B. et al., Phys. Rev. Lett., 102 (2009) 132001.

[6] ABE K. et al., hep-ex/0505037 preprint (2005).

[7] Aubert B. et al., Phys. Rev. Lett., 93 (2004) 041801.

[8] Cotugno G., Faccini R., Polosa A. D. and Sabelli C., Phys. Rev. Lett., 104 (2010) 132005.

[9] Ablikim M. et al., Phys. Lett. B, 656 (2007) 30.

[10] Mizuk R. et al., Phys. Rev. D, 78 (2008) 072004.

[11] Chen K.-F. et al. (Belle Collaboration), Phys. Rev. Lett., 100 (2008) 112001.

[12] Aubert B. et al., Phys. Rev. Lett., 102 (2009) 012001. 